\title{
A CHARACTERIZATION OF $p$-REDUCTIVE LIE ALGEBRAS ${ }^{1}$
}

\author{
ROBERT LEE WILSON
}

\begin{abstract}
Let $\mathfrak{L}$ be a finite dimensional restricted Lie algebra over a field of characteristic $p>0$. Then $\mathfrak{L}$ has a completely reducible faithful finite dimensional restricted representation if and only if $\mathfrak{L}$ has no nonzero nil ideals.
\end{abstract}

The finite dimensional Lie algebras possessing completely reducible faithful finite dimensional representations have been characterized by Jacobson, both over fields of characteristic zero [2] and over fields of prime characteristic [3]. In [4, pp. 98, 99] the similar question is raised of characterizing the finite dimensional restricted Lie algebras over a field of characteristic $p>0$ possessing completely reducible faithful finite dimensional restricted representations. We call such Lie algebras p-reductive. A characterization of these algebras is given by:

THEOREM. Let $\mathfrak{L}$ be a finite dimensional restricted Lie algebra over a field of characteristic $p>0$. Then $\mathfrak{L}$ is $p$-reductive if and only if $\mathfrak{L}$ contains no nonzero nil ideals.

(A subset $S \subseteq \mathfrak{L}$ is said to be $n i l$ if for every $x \in S$ there is some positive integer $n(x)$ such that $x^{p^{n(x)}}=0$.)

Proof. Let $\mathfrak{U}$ be the restricted enveloping algebra of $\mathfrak{L}$ and $\mathfrak{R}$ be the radical of $\mathfrak{U}$. Since $\mathfrak{L}$ generates $\mathfrak{U}$ the map $\Gamma \rightarrow \Gamma \mid \mathfrak{L}$ is a one-one map of the representations of $\mathfrak{U}$ onto the representations of $\mathfrak{L}$ and $\Gamma$ is completely reducible if and only if $\Gamma \mid \mathfrak{L}$ is. Since a representation $\Gamma$ of $\mathfrak{U}$ is completely reducible if and only if ker $\Gamma \supseteq \Re$ we see that $\mathfrak{L}$ is $p$-reductive if and only if $\mathfrak{R} \cap \mathfrak{L}=(0)$. Since $\mathfrak{R} \cap \mathfrak{Q}$ is clearly a nil ideal of $\mathfrak{L}$ which contains every nil ideal, the theorem is proved.

Let $\mathfrak{S}$ denote the solvable radical of $\mathfrak{L}$. Since every nil ideal of $\mathfrak{L}$ is contained in $\mathfrak{S}$ we have:

Corollary. If $\mathfrak{S}$ is p-reductive so is $\mathfrak{L}$.

Presented to the Society, January 21, 1971; received by the editors May 11, 1971.

AMS 1970 subject classifications. Primary 17B20.

Key words and phrases. Completely reducible restricted representations.

${ }^{1}$ The author has received partial support from a Sloan Foundation grant. 
REMARKs. (1) The converse of the Corollary is not true. For example, if $\mathfrak{L}$ is any finite dimensional semisimple restricted Lie algebra and $\mathfrak{W}$ is an irreducible restricted $\mathfrak{L}$ module with basis $\left\{m_{i} \mid 1 \leqq i \leqq r\right\}, r \geqq 2$, we may give the semidirect sum $\mathfrak{L}+\mathfrak{B}$ the structure of a restricted Lie algebra by setting $m_{i}^{p}=m_{1}$ for all $i, 1 \leqq i \leqq r$. Then $\mathfrak{B}$ is the solvable radical of $\mathfrak{L}+\mathfrak{B}$, $\mathfrak{W}$ is not $p$-reductive (for $m_{2}-m_{1}$ spans a nil ideal), and $\mathfrak{L}+\mathfrak{B}$ is $p$-reductive.

(2) Hochschild has shown [1] that every faithful finite dimensional restricted representation of $\mathfrak{L}$ is completely reducible if and only if $\mathfrak{L}$ is abelian and the map $x \rightarrow x^{p}$ is one-one. Thus every nonabelian $p$-reductive Lie algebra has a faithful finite dimensional restricted representation which is not completely reducible.

\section{REFERENCES}

1. G. Hochschild, Representations of restricted Lie algebras of characteristic p, Proc. Amer. Math. Soc. 5 (1954), 603-605. MR 16, 562.

2. N. Jacobson, Rational methods in the theory of Lie algebras, Ann. of Math. (2) 36 (1935), 875-881.

3. - A note on Lie algebras of characteristic p, Amer. J. Math. 74 (1952), 357-359. MR 13, 816.

4. G. B. Seligman, Modular Lie algebras, Ergebnisse der Mathematik und ihrer Grenzgebiete, Band 40, Springer-Verlag, New York, 1967. MR 39 \#6933.

Courant Institute of Mathematical Sciences, New York University, New YORK, NEW YORK 10012

Current address: Department of Mathematics, Rutgers University, New Brunswick, New Jersey 08903 